\title{
MS081.P01
}

\section{Poster Presentation}

UgpB protein dominantly follows Sec translocation pathway

Angshu Dutta ${ }^{1}$, Shankar Prasad Kanaujia ${ }^{1}$

${ }^{1}$ Dept. of Biosciences and Bioengineering, Indian Institute of Technology Guwahati, Guwahati, India

E-mail: angshudutta39@iitg.ernet.in

Microorganisms secrete proteins to carry out different types of biological functions including biogenesis of flagella or pili, sequestration of nutrients, pathogenicity, etc. Translocation of these proteins across the plasma membrane to the periplasm or external environment takes place via two main pathways viz. Sec (secretory) and Tat (twin arginine translocation). Sec pathway translocates protein in their unfolded state, whereas, Tat pathway favors the translocation of folded proteins. However, the selection of a particular translocation pathway must be accomplished during or immediately after the completion of protein biosynthesis. This suggests that the primary structure of proteins itself must have some specific signatures for Sec- and Tat-mediated translocation. To identify and further verify the signature motifs and properties of proteins translocated through Sec and Tat pathways, we took up a case study of periplasmic UgpB protein. UgpB is a periplasmic solute (or substrate) binding protein of UgpABCE ATP-binding cassette (ABC) transporter, which mediates the sequestration of either sn-glycerol-3-phosphate (G3P) or glycerophosphocholine (GPC) molecules in the periplasmic space. Recently, UgpB protein from Mycobacterium tuberculosis (MtUgpB) was reported to follow the Tat pathway for its translocation to the periplasm during its pathogenesis; hence it has been proposed as a promising candidate for drug target. Thus, in this study, we attempted to identify the translocation pathway for all UgpB proteins from different organisms available in protein databases. Our computational analysis of these proteins reveals that most of the UgpB proteins follow Sec pathway rather than Tat pathway, unlike MtUgpB. Moreover, this study reveals that the selection of pathway by UgpB proteins mainly depends on the characteristics of signal peptide. In addition, it suggests that the selection of the translocation pathway can also be influenced by the environment in which the organism thrives.

[1] Natale, P. et al. (2008) Biochim. Biophys. Acta. 1778, 1735-1756.

[2] Jiang, D. et al. (2014) FEBS J. 281, 331-341.

[3] Chandravanshi, M. et al. (2016) Gene 592, 260-268.

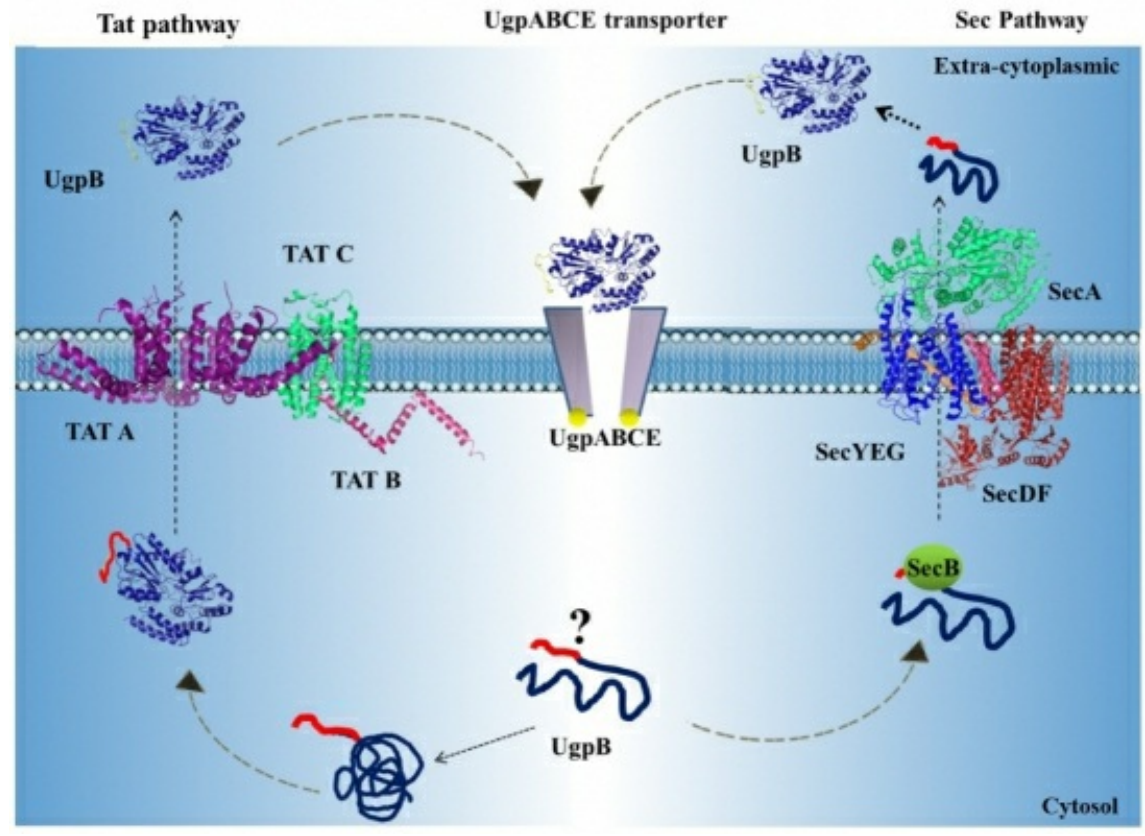

Keywords: UgpB, Signal peptide, Sec pathway 